CYBERNETICS AND INFORMATION TECHNOLOGIES • Volume 16, No 5

Special Issue on Application of Advanced Computing and Simulation in Information Systems

Sofia $\bullet 2016$

Print ISSN: 1311-9702; Online ISSN: 1314-4081

DOI: $10.1515 /$ cait-2016-0061

\title{
Capacity of Right-Turn Lane at Signalized Intersection under Pedestrian-Bicycle Effect
}

\author{
Chao Gao, Xiao-Ming Liu, Xiao-Kuan Yang
}

Beijing Key Laboratory of Traffic Engineering, Beijing University of Technology, Beijing 100124, China

Emails: gaochao@emails.bjut.edu.cn_1248169988@qq.comｘiaokuan@hotmail.com

\begin{abstract}
The effect of pedestrian and bicycle infrastructure is used to analyze the capacity for right-turn lane at signalized intersection. Each cycle at the signalized intersection was divided into several periods. The effect of pedestrian and bicycle at each period was analyzed. The number of right-turn cars going through signalized intersection was calculated by using probability theory and mathematical statistics. The capacity under the effect of pedestrian and bicycle for right-turn lane at signalized Intersection was deduced. The calculated results were deduced by using the survey data of two signalized intersections at Jinan city. The mean difference values are $8 \%$ and $10.1 \%$, compared with VISSIM simulation results. The comparisons show that this model can fully describe the effect of pedestrian and bicycle to signalized intersection.
\end{abstract}

Keywords: Traffic engineering, Capacity, Pedestrian-bicycle, Signalized intersection, Right-turn lane.

\section{Introduction}

When there are heavy pedestrian and bicycle flows at a signalized intersection, the conflicts between pedestrian-bicycle flow and vehicle flow at right-turn lane are obvious. The vehicle flow is usually blocked by the pedestrian-bicycle flow crosswise, and the effect for the capacity of vehicle flow at right-turn lane is significant. A lot of factors are posed in HCM2010 [1] to describe the influence for the capacity of right-turn lane, such as signal timing, traffic composition, lane width, etc. Code for the design of urban road engineering method [2] and stop line method [3] make systematical description of the calculation method at a signalized intersection, and simply describe the impact of the pedestrian-bicycle flow to the vehicle flow at right-turn lane. Many studies [2, 4-8] focus on this impact, and many methods are used to deduce the capacity models, such as data fitting method 
$[5,8]$, gap acceptance theory [6, 7, 9], traffic flow wave theory [10] and mathematical statistics method [11]. In order to deduce capacity models, the concepts of bicycle group [6] and pedestrian group [7] were proposed separately to describe the distribution pedestrian and bicycle flows. Study [8] divides the vehiclebicycle conflict at intersections into several units by using temporal separation and spatial separation method.

This paper focuses on the impacts of pedestrian-bicycle flow for right-turn lane at normal signalized intersection. Signal cycle is divided into several periods, and capacity model is developed. The calculated values of model are verified by VISSIM simulation results.

\section{Conflicts between pedestrian-bicycle flow and vehicle flow}

In the space domain, the transverse interference of right-turn vehicle flow is from the pedestrian-bicycle flow at an intersection entrance and an exit. When it is at a green phase, the agglomerated pedestrian and bicycle flows at pedestrian-bicycle stop line will continuously cross right-turn lane, and the vehicle flow will be blocked until all the agglomerated pedestrian and bicycle flows getting through. Fig. 1 is the schematic diagram of a normal intersection. The right-turn vehicle flow from southern entrance should pass through the pedestrian-bicycle flow of westward, eastward, northward and southward.

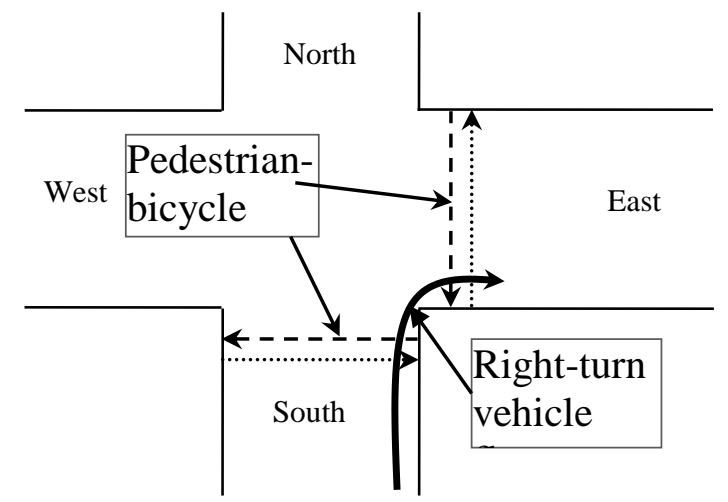

Fig. 1. Diagram of right-turn vehicle flow passing through bicycle-pedestrian flow

In the time domain, setting of bicycle-pedestrian phase usually accords to the vehicle phase. Therefore, east-westward and north-southward bicycle-pedestrian flows will cross through the intersection at different phases.

Taking east-westward phase for example, bicycle and pedestrian flows which cross the intersection can be illustrated as schematic diagram of Fig. 2. The agglomerated bicycle-pedestrian flow waits at western stop line until the signal indication changing to green, then go cross the stop line continuously. Hypothesize this time-period lasts HI. These flows spread to bicycle flow and pedestrian flow when the flows arrive to the right-turn lane at southern entrance. Hypothesize the time-period of bicycle flow going through right-turn lane lasts $\mathrm{CD}$, and pedestrian flow lasts EF. The bicycle flow and pedestrian flow of these time-periods will have 
a block effect for right-turn vehicle flow. Hypothesize the westward bicyclepedestrian flow going through right-turn lane lasts time-period $\mathrm{AB}$. And it will have a block effect for right-turn vehicle flow too. BC, DE and FG are the time-periods of stochastic bicycle-pedestrian flow going through right-turn lane.

Because the conflict mechanisms of the flows at entrance and exit are almost the same, Fig. 2 can describe the conflict mechanism of flows at exit too.

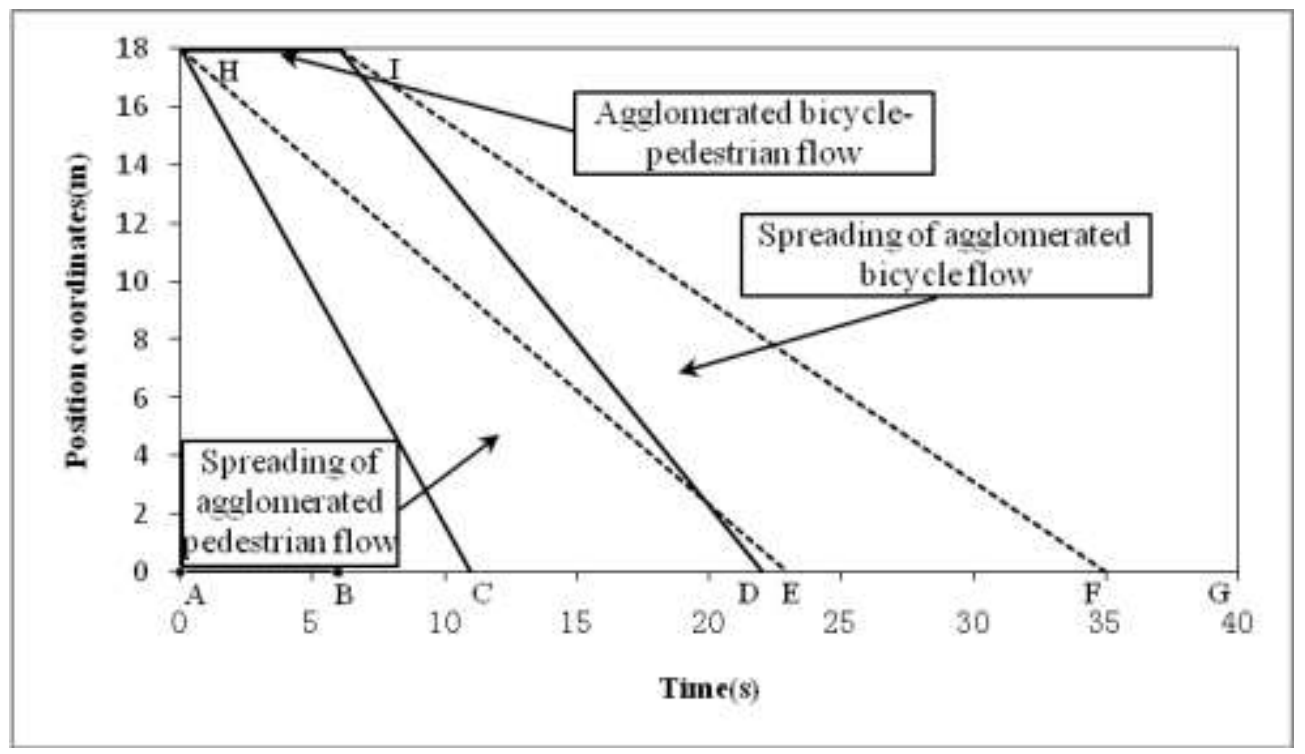

Fig. 2. Spreading of bicycle- pedestrian flow

\section{Modelling}

There are five hypothesizes of this paper: (1) there is one right-turn lane at each typical intersection, (2) all the drivers and pedestrians obey the traffic law, (3) the intersection is once-cross street intersection, (4) the green times of bicycle and pedestrian are simplified as equal to the green time of vehicle, (5) other influencing factors are not considered.

\subsection{Capacity model}

The signal cycle time can be divided into three periods. And the capacity model of right-lane can be hypothesized as the following formula (1).

$$
C=\frac{3600}{T}\left(N_{\mathrm{EW}}+N_{\mathrm{SN}}+N_{\mathrm{R}}\right),
$$

where $T$ is signal cycle time, $N_{\mathrm{Ew}}$ is the number of vehicles going through at the east-westward green time of one signal cycle, $N_{\mathrm{SN}}$ is the number of vehicles going through at the north-southward green time of one signal cycle, $N_{\mathrm{R}}$ is the number of 
vehicles going through at the red time of one signal cycle. And, $N_{\mathrm{R}}$ can be calculated by formula (2).

$$
N_{\mathrm{R}}=\frac{T-T_{\mathrm{SN}}-T_{\mathrm{EW}}-T_{\mathrm{L}}}{\overline{H_{t}}},
$$

where $T_{\mathrm{SN}}$ is the north-southward green time, $T_{\mathrm{EW}}$ is the east-westward green time, $\overline{H_{t}}$ is the headway of vehicle flow at right-lane, $T_{\mathrm{L}}$ is lost time.

\section{2. $N_{\mathrm{SN}}$ and $N_{\mathrm{EW}}$ models}

The number of vehicles going through at north-southward green time is the sum of vehicle going through at $\mathrm{AB}, \mathrm{BC}, \mathrm{CD}, \mathrm{DE}, \mathrm{EF}$ and FG periods. Parameter $N_{\mathrm{SN}}$ can be calculated as

$$
N_{\mathrm{EW}}=N_{b \mathrm{EW}}+N_{p \mathrm{EW}}+N_{\mathrm{EW} 1}+N_{\mathrm{EW} 2}+N_{\mathrm{EW} 3},
$$

where $N_{b \mathrm{EW}}$ is the number of vehicles going through a stochastic flow of bicyclepedestrian at CD period, $N_{p \mathrm{EW}}$ is the number of vehicles going through a stochastic flow of bicycle-pedestrian at $\mathrm{EF}$ period, $N_{\mathrm{EW} 1}$ is the number of vehicles going through the gap between bicycle flow and pedestrian flow at BC period, $N_{\mathrm{EW} 2}$ is the number of vehicles going through the gap at DE period, $N_{\mathrm{EW} 3}$ is the number of vehicles going through at surplus time after agglomerated pedestrian flow.

In the same way, $N_{\mathrm{SN}}$ can be deduced $b$ using the abovementioned method. The following is the deduction of variables in (3).

3.3. Number of vehicles going through under conflicts with multi-bicycle and pedestrian flows

Let's assume that the arrival distributions of bicycle flows and pedestrian flows obey the Poisson distribution [8]. And the bicycles or pedestrians can overtake freely. Hypothesizing the arrival rate is $\lambda$, the distribution function is $F(t)=1-\operatorname{Exp}(-\lambda t)$. The probability of headway more than $t$ (minutes) is $f(t)=P(h t>t)=\operatorname{Exp}(\lambda t)$. Hypothesizing there are two independent flows, their arrival rate are $\lambda_{1}$ and $\lambda_{2}$. The following formula can be deduced from conditional probability:

$$
f_{12}(t)=f_{1}(t) \cdot f_{2}(t)=\operatorname{Exp}\left(-\lambda_{1} t\right) \cdot \operatorname{Exp}\left(-\lambda_{2} t\right)=\operatorname{Exp}\left[-\left(\lambda_{1}+\lambda_{2}\right) t\right] .
$$

Therefore, when two flows mixed to one flow, the mixed flow still obeys Poisson distribution with the arrival rate of $\lambda_{1}+\lambda_{2}$.

Let's assume that a certain bicycle-pedestrian flow obeys Poisson distribution. The duration time is $T$. The headway of right-turn vehicle flow is $\overline{H_{t}}$. The critical gap vehicle flow going through bicycle-pedestrian flow is $t_{0}$. Subsection 
summation method is used to derive the number of right-turn vehicles going through at time segment $T$.

If $0<t<t_{0}$, there is 0 vehicle going through. The probability is $F(0)=1-\operatorname{Exp}\left(-\lambda t_{0}\right)$. The frequency is $N_{0}=\lambda T \cdot F(0)=\lambda T \cdot\left[1-\operatorname{Exp}\left(-\lambda t_{0}\right)\right]$.

If $t_{0}+(i-1) \cdot \overline{H_{t}}<t<t_{0}+i \cdot \overline{H_{t}}$, there is $i$ vehicles going through. The probability is $F(i)=\operatorname{Exp}\left\{-\lambda\left[t_{0}+(i-1) \overline{H_{t}}\right]\right\}-\operatorname{Exp}\left[-\lambda\left[t_{0}+i \cdot \overline{H_{t}}\right] . \quad\right.$ The frequency is

$$
N_{i}=\lambda T \cdot F(i)=\lambda T \cdot \operatorname{Exp}\left\{-\lambda\left[t_{0}+(i-1) \overline{H_{t}}\right]\right\}-\lambda T \cdot \operatorname{Exp}\left[-\lambda\left[t_{0}+i \cdot \overline{H_{t}}\right]\right],
$$

where $i=1,2, \ldots, n$.

The number of right-turn vehicles going through at time segment $T$ is deduced to formula

$$
\begin{gathered}
N=\sum_{i=0}^{n}\left(N_{i} \cdot i\right)=\sum_{i=0}^{n}(\lambda T \cdot F(i) \cdot i)= \\
=\lambda T \cdot\left\{\sum_{i=0}^{n} \operatorname{Exp}\left[-\lambda t_{0}+(i-1) \overline{H_{t}}\right]-n \cdot \operatorname{Exp}\left(-\lambda t_{0}+n \cdot \overline{H_{t}}\right)\right\} .
\end{gathered}
$$

That formula, by using the method of sum of the geometric progression can be deduced to

$$
N=\lambda T \cdot\left\{\operatorname{Exp}\left(-\lambda t_{0}\right) \frac{1-\operatorname{Exp}\left[-\lambda(n-1) \overline{H_{t}}\right]}{1-\operatorname{Exp}\left(-\lambda \overline{H_{t}}\right)}-n \cdot \operatorname{Exp}\left(-\lambda t_{0}+n \cdot \overline{H_{t}}\right)\right\} .
$$

Let be $n \rightarrow \infty$, formula (5) can be deduced to

$$
N=\frac{\lambda T \cdot \operatorname{Exp}\left(-\lambda t_{0}\right)}{1-\operatorname{Exp}\left(-\lambda \overline{H_{t}}\right)}
$$

Let's assume that the volumes of bicycle flow and pedestrian flow westward and eastward are $Q_{b \mathrm{WE}}, Q_{p \mathrm{WE}}, Q_{b \mathrm{EW}}$ and $Q_{p \mathrm{EW}}$,corresponding to the arrival rates as $\lambda_{b \mathrm{WE}}, \lambda_{p \mathrm{WE}}, \lambda_{b \mathrm{EW}}$ and $\lambda_{p \mathrm{EW}}$. When the agglomerated bicycle flow and pedestrian flow arrive eastward to right-turn lane of southern entrance, the arrival rates are $\lambda_{b \mathrm{WE}}^{\prime}$ and $\lambda_{p \mathrm{WE}}^{\prime}$. The relationship between $\lambda$ and $Q$ is $\lambda=Q / 3600$.

Parameters $N_{b \mathrm{EW}}, N_{\mathrm{EW} 1}, N_{\mathrm{EW} 2}$ and $N_{\mathrm{EW} 3}$ can be calculated by

$$
\begin{gathered}
N_{b \mathrm{EW}}=\frac{\left(\lambda_{b \mathrm{WE}}^{\prime}+\lambda_{b \mathrm{EW}}+\lambda_{p \mathrm{EW}}\right) \cdot T_{\mathrm{CD}} \cdot \operatorname{Exp}\left[-\left(\lambda_{b \mathrm{WE}}^{\prime}+\lambda_{b \mathrm{EW}}+\lambda_{p \mathrm{EW}}\right) t_{0}\right]}{1-\operatorname{Exp}\left[-\left(\lambda_{b \mathrm{WE}}^{\prime}+\lambda_{b \mathrm{EW}}+\lambda_{p \mathrm{EW}}\right) \overline{H_{t}}\right]}, \\
N_{p \mathrm{EW}}=\frac{\left(\lambda_{p \mathrm{WE}}^{\prime}+\lambda_{b \mathrm{EW}}+\lambda_{p \mathrm{EW}}\right) \cdot T_{\mathrm{EF}} \cdot \operatorname{Exp}\left[-\left(\lambda_{p \mathrm{WE}}^{\prime}+\lambda_{b \mathrm{EW}}+\lambda_{p \mathrm{EW}}\right) t_{0}\right]}{1-\operatorname{Exp}\left[-\left(\lambda_{p \mathrm{WE}}^{\prime}+\lambda_{b \mathrm{EW}}+\lambda_{p \mathrm{EW}}\right) \overline{H_{t}}\right]}, \\
N_{\mathrm{EW} 1}=\frac{\left(\lambda_{b \mathrm{EW}}+\lambda_{p \mathrm{EW}}\right) \cdot T_{\mathrm{BC}} \cdot \operatorname{Exp}\left[-\left(\lambda_{b \mathrm{EW}}+\lambda_{p \mathrm{EW}}\right) t_{0}\right]}{1-\operatorname{Exp}\left[-\left(\lambda_{b \mathrm{EW}}+\lambda_{p \mathrm{EW}}\right) \overline{H_{t}}\right]}
\end{gathered}
$$


(10)

$$
\begin{gathered}
N_{\mathrm{EW} 2}= \\
=\frac{\left(\lambda_{b \mathrm{WE}}+\lambda_{p \mathrm{WE}}+\lambda_{b \mathrm{EW}}+\lambda_{p \mathrm{EW}}\right) \cdot T_{\mathrm{DE}} \cdot \operatorname{Exp}\left[-\left(\lambda_{b \mathrm{WE}}+\lambda_{p \mathrm{WE}}+\lambda_{b \mathrm{EW}}+\lambda_{p \mathrm{EW}}\right) t_{0}\right]}{1-\operatorname{Exp}\left[-\left(\lambda_{b \mathrm{WE}}+\lambda_{p \mathrm{WE}}+\lambda_{b \mathrm{EW}}+\lambda_{p \mathrm{EW}}\right) \overline{H_{t}}\right]} \\
N_{\mathrm{EW} 3}=
\end{gathered}
$$

$$
=\frac{\left(\lambda_{b \mathrm{WE}}+\lambda_{p \mathrm{WE}}+\lambda_{b \mathrm{EW}}+\lambda_{p \mathrm{EW}}\right) \cdot T_{\mathrm{FG}} \cdot \operatorname{Exp}\left[-\left(\lambda_{b \mathrm{WE}}+\lambda_{p \mathrm{WE}}+\lambda_{b \mathrm{EW}}+\lambda_{p \mathrm{EW}}\right) t_{0}\right]}{1-\operatorname{Exp}\left[-\left(\lambda_{b \mathrm{WE}}+\lambda_{p \mathrm{WE}}+\lambda_{b \mathrm{EW}}+\lambda_{p \mathrm{EW}}\right) \overline{H_{t}}\right]},
$$

where $T_{\mathrm{AB}}, T_{\mathrm{BC}}, T_{\mathrm{CD}}, T_{\mathrm{DE}}, T_{\mathrm{EF}}$ and $T_{\mathrm{FG}}$ are the duration times of time segments $\mathrm{AB}, \mathrm{BC}, \mathrm{CD}, \mathrm{DE}, \mathrm{EF}$ and $\mathrm{FG}$.

\subsection{Arrival rates of agglomerated flow and time segments}

Let's assume that the speeds at the front of agglomerated bicycle flow and pedestrian flow are $V_{b 1}$ and $V_{p 1}$. The speeds at the end of agglomerated bicycle flow and pedestrian flow are $V_{b 2}$ and $V_{p 2}$. The length of bicycle lane and crosswalk is $L$, the widths are $W_{b}$ and $W_{p}$. The numbers of agglomerated bicycles and pedestrians waiting at eastern stop line at the end of red signal are $N_{b \mathrm{E}}$ and $N_{p \mathrm{E}}$. The numbers of agglomerated bicycles and pedestrians waiting at western stop line at the end of red signal are $N_{b \mathrm{~W}}$ and $N_{p \mathrm{~W}}$.

Parameters $N_{b \mathrm{E}}, N_{p \mathrm{E}}, N_{b \mathrm{~W}}$ and $N_{p \mathrm{~W}}$ can be calculated by:

$$
\begin{aligned}
& N_{b \mathrm{E}}=Q_{b \mathrm{EW}}\left(T-T_{\mathrm{EW}}\right) / T, \\
& N_{p \mathrm{E}}=Q_{p \mathrm{EW}}\left(T-T_{\mathrm{EW}}\right) / T, \\
& N_{b \mathrm{~W}}=Q_{b \mathrm{WE}}\left(T-T_{\mathrm{WE}}\right) / T, \\
& N_{p \mathrm{~W}}=Q_{p \mathrm{WE}}\left(T-T_{\mathrm{WE}}\right) / T .
\end{aligned}
$$

Let's assume that the duration times of the agglomerated bicycles and pedestrians going westward and eastward are $T_{b \mathrm{HI}}, T_{p \mathrm{HI}}, T_{b \mathrm{AB}}$ and $T_{p \mathrm{AB}}$. Hypothesize the duration time is proportional to volume and inversely proportional to lane width.

Parameters $T_{b \mathrm{HI}}, T_{p \mathrm{HI}}, T_{b \mathrm{AB}}$ and $T_{p \mathrm{AB}}$ can be calculated by:

$$
\begin{gathered}
T_{b \mathrm{HI}}=a_{b 1} \cdot Q_{b \mathrm{WE}}\left(T-T_{\mathrm{WE}}\right) /\left(\mathrm{TW}_{b}\right), \\
T_{p \mathrm{HI}}=a_{p 1} \cdot Q_{p \mathrm{WE}}\left(T-T_{\mathrm{WE}}\right) /\left(\mathrm{TW}_{p}\right), \\
T_{b \mathrm{AB}}=a_{b 1} \cdot Q_{b \mathrm{AB}}\left(T-T_{\mathrm{EW}}\right) /\left(\mathrm{TW}_{b}\right), \\
T_{p \mathrm{AB}}=a_{p 1} \cdot Q_{p \mathrm{AB}}\left(T-T_{\mathrm{EW}}\right) /\left(\mathrm{TW}_{p}\right) .
\end{gathered}
$$

The coordinate values of points $\mathrm{C}, \mathrm{D}, \mathrm{E}$ and $\mathrm{F}$ can be calculated by:

$$
T_{\mathrm{C}}=L / V_{b 1} \text {, }
$$




$$
\begin{gathered}
T_{\mathrm{D}}=T_{b \mathrm{HI}}+L / V_{b 2}=a_{b 1} \cdot Q_{b \mathrm{WE}}\left(T-T_{\mathrm{WE}}\right) /\left(\mathrm{TW}_{b}\right)+L / V_{b 2}, \\
T_{\mathrm{E}}=L / V_{p 1}, \\
T_{\mathrm{F}}=T_{p \mathrm{HI}}+L / V_{p 2}=a_{p 1} \cdot Q_{p \mathrm{WE}}\left(T-T_{\mathrm{WE}}\right) /\left(\mathrm{TW}_{p}\right)+L / V_{p 2} .
\end{gathered}
$$

And the lengths of $\mathrm{AB}, \mathrm{BC}, \mathrm{CD}, \mathrm{DE}, \mathrm{EF}$ and $\mathrm{FG}$ can be calculated by:

$$
\begin{gathered}
T_{\mathrm{AB}}=\operatorname{Max}\left\{T_{p \mathrm{AB}}, T_{b \mathrm{AB}}\right\}, \\
T_{\mathrm{BC}}=L / V_{b 1}-a_{b 1} \cdot Q_{b \mathrm{AB}}\left(T-T_{\mathrm{EW}}\right) /\left(\mathrm{TW}_{b}\right), \\
T_{\mathrm{CD}}=a_{b 1} \cdot Q_{b \mathrm{WE}}\left(T-T_{\mathrm{WE}}\right) /\left(\mathrm{TW}_{b}\right)+L / V_{b 2}-L / V_{b 1}, \\
T_{\mathrm{DE}}=L / V_{p 1}-L / V_{b 2}-a_{b 1} \cdot Q_{b \mathrm{WE}}\left(T-T_{\mathrm{WE}}\right) /\left(\mathrm{TW}_{b}\right), \\
T_{\mathrm{EF}}=a_{p 1} \cdot Q_{p \mathrm{WE}}\left(T-T_{\mathrm{WE}}\right) /\left(\mathrm{TW}_{p}\right)+L / V_{p 2}-L / V_{p 1} .
\end{gathered}
$$

The arrival rates of eastward agglomerated flow arriving at right-turn lane can be calculated by:

$$
\begin{aligned}
& \lambda_{b \mathrm{WE}}^{\prime}=\lambda_{b \mathrm{WE}} \cdot T_{b \mathrm{HI}} / T_{b \mathrm{CD}}, \\
& \lambda_{p \mathrm{WE}}^{\prime}=\lambda_{p \mathrm{WE}} \cdot T_{p \mathrm{HI}} / T_{p \mathrm{EF}} .
\end{aligned}
$$

\subsection{Special cases}

(1) If the crosswalk is very short, the condition of $T_{\mathrm{B}}>T_{\mathrm{C}}$ may appear. The time series in turn are $\mathrm{AC}, \mathrm{CB}, \mathrm{BD}, \mathrm{DE}, \mathrm{EF}$ and FG. The agglomerated flow will block the right-turn vehicles at time segments $\mathrm{AC}$ and $\mathrm{CB}$. The calculation method after time point B obeys Section 3.4.

(2) If the crosswalk is very short, or the bicycle-pedestrian flow is heavy, the condition of $T_{\mathrm{D}}>T_{\mathrm{E}}$ may appear. The time series in turn are AB, BC, CE, ED, DF and FG. The arrival rate of bicycle-pedestrian flow at time segment ED is $\lambda_{b \mathrm{WE}}^{\prime}+\lambda_{p \mathrm{WE}}^{\prime}+\lambda_{b \mathrm{EW}}+\lambda_{p \mathrm{EW}}$. The segment times of $\mathrm{CE}, \mathrm{ED}$ and DF should be recalculated.

(3) If $T_{\mathrm{F}}>T_{\mathrm{EW}}$, the green signal can't meet the demand of pedestrian flow to go through crosswalk. Twice-cross street should be considered for signal timing.

\section{Example and simulation verification}

Basic data is collected by field survey at Jingshi Road\& Nanxinzhuangxi Road intersection Jiluo Road\& Beiyuan Road intersection of Jinan city, China. And the capacity values are calculated by using the basic data. Capacity value is hard to acquire by field survey, so VISSIM simulation is used to verify the calculating results. The simulation diagram is shown in Fig. 3. 


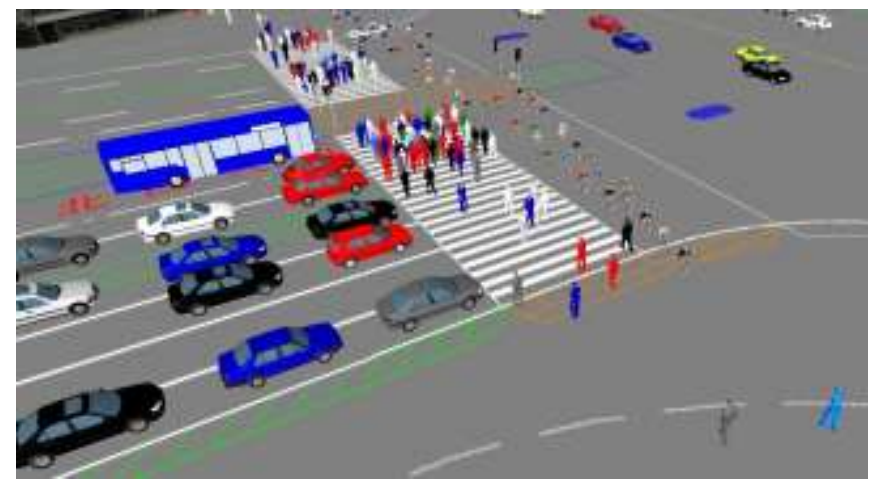

Fig. 3. Simulation diagram

To compare calculated values with simulated values is used the next formula:

$$
R_{\mathrm{D}}=\sum_{i=1}^{10}\left|S_{i}-C_{i}\right| / \sum_{i=1}^{10} C_{i}
$$

where $R_{\mathrm{D}}$ is the mean deviation ratio, $S_{i}$ is simulated value, $i$ is the sample number, $C_{i}$ is calculated value. The comparison diagrams are shown at Fig. 4 and Fig. 5.

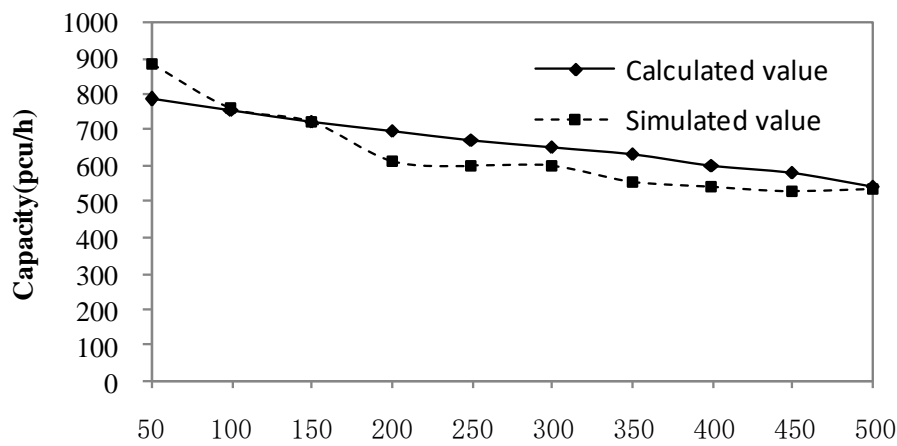

Volume of bicycle- pedestrian flow (pcu/h)

Fig. 4. Comparison between calculated value and simulation value at Jingshi Road \& Nanxinzhuangxi Road intersection 


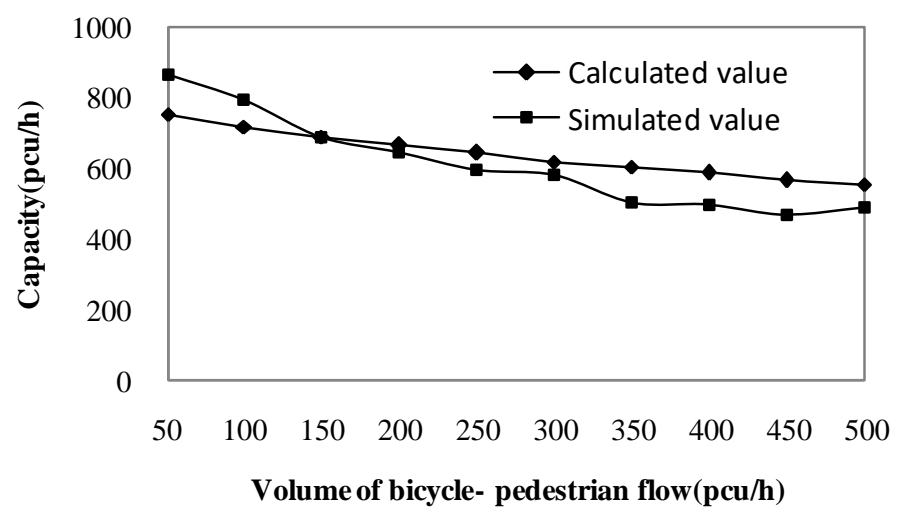

Fig. 5. Comparison between calculated value and simulation value at Jiluo Road \& Beiyuan Road intersection

The $R_{\mathrm{D}}$ value of Jingshi Road \& Nanxinzhuangxi Road intersection is $8 \%$, and the $R_{\mathrm{D}}$ value of Jiluo Road \& Beiyuan Road intersection is $10.1 \%$. The calculated value fits simulated value very well. Therefore, the method is suitable to calculate the capacity of right-turn lane.

\section{Conclusion}

(1) This study focus on the conflicts between pedestrian-bicycle flow and vehicle flow at right-turn lane, and puts forward the capacity model right-turn lane. It provides the theoretical basis for the studies of capacity under heavy pedestrianbicycle flow.

(2) If the length of crosswalk is very long or the volume of pedestrian flow is heavy, it is hard for once-cross street. Twice-cross street should be considered for signal timing.

(3) There are many influencing factors for the driving of right-turn vehicle. One right-turn lane at typical intersection is considered in the paper. The scholars can focus on the modifications of lane width, traffic composition, and multiple right lanes.

Acknowledgements: This study was supported by 973 Program (2012CB723303), Science\& Technology Project of Shandong Province (J14LN78), Foundation of Shandong Jiaotong University (Z201406).

\section{References}

1. National Research Council. Highway Capacity Manual. Washington DC., Transportation Research Board, 2010.

2. CJJ 37-2012. Code for Design of Urban Road Engineering. 2012. 
3. X u, J. Q., X. W. C h e n. Traffic Engineering. 3rd Edition. Beijing, China Communications Press, 2008.

4. Li u, G. X., K. P. L i, Y. N i. An Overview on Pedestrian Psychology and Behavior when Crossing Intersections. - Technology \& Economy in Areas of Communication, Vol. 5, 2008, No 49, pp. 159-166.

5. M a, Z. D., X. M. Y a n g. A Study on the Capacity of Exclusive Right-Turn Lanes of At-Grade Intersections. - China Civil Engineering Journal, Vol. 39, 2006, No 12, pp. 108-111.

6. Z h a o, J., Y. B a i, X. G. Y a n g. A Regression-Based Pedestrian-Bicycle Adjustment Factor for Capacity of Right-Turn Movements. - Journal of Highway and Transportation Research and Development, Vol. 29, 2012, No 2, pp. 120-126.

7. S h i, Y. C. An Exploration of Modeling of Right-Turn Line Capacity at Signalized Intersection. Beijing University of Technology, Vol. 5, 2007, No 15, pp. 21-53.

8. Li u, Y. L. A Study of Right-Turn Lane Capacity Influenced by Pedestrians at Signalized Intersections. - Road Traffic\& Safety, Vol. 10, 2015, No 15, pp. 1-5.

9. Lai, Y. W., J. Rong, X. M. Li u. Capacity Model of the Right-Turn Lane at Signalized Intersection with Channelized Islands. - Journal of Beijing University of Technology, Vol. 38, 2012, No 6, pp. 865-869.

10. Q i a n, D. L., Z. Q. N i u. Analysis of Capacity and Its Sensitivity of Right-Turn Motor Vehicles at Signalized Intersection under Mixed Traffic. - China Journal of Highway and Transport, Vol. 2, 2010, No 2, pp. 77-83.

11. La i, Y. W., J. R on g, X. M. Li u. Through Capacity of Signalized Intersection Influenced by Short Right Turn Lane. - Journal of Highway and Transportation Research and Development, Vol. 30, 2013, No 3, pp. 131-135. 